\title{
Erratum: Superconductivity in $\mathrm{CoO}_{2}$ Layers and the Resonating Valence Bond Mean Field Theory of the Triangular Lattice t-J Model [Phys. Rev. B 68 , 104508 (2003)]
}

\author{
Brijesh Kumar ${ }^{1,2}$ and B. Sriram Shastry ${ }^{1,3}$ \\ (1)Department of Physics, Indian Institute of Science, Bangalore 560012, India. \\ (2) ITP, École Polytechnique Fédérale de Lausanne, CH 1015 Lausanne Switzerland \\ (3) Department of Physics, University of California Santa Cruz, Ca 95064
}

Our recent paper [1] contains an error in the formula for the high frequency Hall constant for the experimentally relevant case of electron doping. $\mathrm{Eq}(3)$ giving the high frequency Hall constant for the case of electron filling, i.e. electron density $n>1$, should correctly read

$$
\left.R_{H}^{*}=-\frac{v}{4|e|} \frac{k_{B} T}{t} \frac{1+\delta}{\delta(1-\delta)} \quad \text { (Cases } \mathrm{A}(\mathrm{i}) \text { or } \mathrm{B}(\mathrm{ii})\right) \quad \mathrm{Eq}\left(3^{\prime}\right)
$$

where $\delta=|1-n|$. This differs essentially from the quoted answer by a minus sign that was missed by us, and by a factor of 2. This equation is obtained from $\mathrm{Eq}(2)$ in the paper, calculated for the case of hole doping $n<1$, by a particle hole transformation. Performing a canonical transformation $c_{i, \sigma} \leftrightarrow c_{i, \sigma}^{\dagger}$ in the Kubo formulas for models of lattice fermions (see e.g. [2] Eqn(1,2) ) yields:

$$
R_{H}(n, t, \omega, T)=-R_{H}(2-n,-t, \omega, T) .
$$

The crucial overall minus sign arises from the fact that the Hall constant involves a three current propagator, and each current picks up a minus sign under the transformation. Incidentally this identity results in a vanishing at half filling for bipartite lattices of the Hall function $R_{H}(1, t, \omega, T)$ for any value of $T, \omega$ and the interaction strength.

The changed sign in $\mathrm{Eq}\left(3^{\prime}\right)$ has a few implications which we would like to state explicitly. The recent experiment of Wang, Rogado, Cava and Ong [3] on the transport Hall constant, actually sees the predicted linear T behaviour over a wide temperature range $200^{\circ} K \leq T \leq 400^{\circ} K$ with a positive slope. From $\mathrm{Eq}\left(3^{\prime}\right)$ above, we deduce that the appropriate $t-J$ model description of the system has $t<0$, i.e. belongs to case $\mathrm{B}(\mathrm{ii})$ in our notation, at least at the composition $x \sim .7$.

We note that with this correction, the sign of the hopping $t$ suggested by our calculation is the same as the one favoured by Refs [4,5], at least for the non superconducting range $x \sim .7$. In this composition range, photoemission data [6,7] also suggest the same sign of $t$, with an unoccupied region around the center of Brillouin zone, namely the $\Gamma$ point.

[1] B Kumar and B S Shastry, Phys Rev B 68 , 104508 (2003) and cond-mat/0304210.

[2] B S Shastry, B I Shraiman and R R P Singh, Phys. Rev. Lett. 70, 2004 (1993).

[3] Y Wang, N S Rogado, R J Cava and N P Ong, cond-mat/0305455.

[4] G Baskaran,Phys. Rev. Lett. 91097003 (2003) and cond-mat/0303649.

[5] Q H Wang,D-H Lee, and P A Lee, cond-mat/0304377.

[6] M Z Hasan, Y D Chuang, A P Kuprin, Y Kong, D Qian, Y W Li, B L Mesler, Z Hussain, A V Fedorov, R Kimmerling, E Rotenberg, K Rossnagel, H Koh, M Rogado, M L Foo and R J Cava, cond-mat/0308438,

[7] H -B Yang,S -C Wang, A K P Sekharan, H Matsui, S Souma, T Sato, T Takahashi, T Takeuchi, J C Campuzano, R Jin, B C Sales, D Mandrus, Z Wang, H Ding, condmat/0310532. 


\title{
Superconductivity in $\mathrm{CoO}_{2}$ Layers and the Resonating Valence Bond Mean Field Theory of the Triangular Lattice t-J Model
}

\author{
Brijesh Kumar and B. Sriram Shastry \\ Department of Physics, Indian Institute of Science, Bangalore 560012, India.
}

\begin{abstract}
Motivated by the recent discovery of superconductivity in two dimensional $\mathrm{CoO}_{2}$ layers, we present some possibly useful results of the RVB mean field theory applied to the triangular lattice. An interesting time reversal breaking superconducting state arises from strongly frustrated interactions. Away from half filling, the order parameter is found to be complex, and yields a fully gapped quasiparticle spectrum. The sign of the hopping plays a crucial role in the analysis, and we find that superconductivity is as fragile for one sign as it is robust for the other. $\mathrm{Na}_{x} \mathrm{CoO}_{2} \cdot y \mathrm{H}_{2} \mathrm{O}$ is argued to belong to the robust case, by comparing the LDA fermi surface with an effective tight binding model. The high frequency Hall constant in this system is potentially interesting, since it is pointed out to increase linearly with temperature without saturation for $\mathrm{T}>\mathrm{T}_{\text {degeneracy }}$.

PACS number: 74.20.Mn, 74.20.Rp, 71.27.+a, 71.18.+y
\end{abstract}

\section{INTRODUCTION}

The recent discovery of superconductivity at low temperatures in $\mathrm{CoO}_{2}$ layered compounds [1] is an exciting event, since it may be the long sought low temperature RVB superconductor, on a lattice which was at the basis of Anderson's original ideas on a possible quantum spin liquid state [2]. Although the spin-1/2 triangular lattice appears to have better states with three sublattice magnetic order, it is possible that the RVB state is attained for sufficiently high doping, and it seems to be both useful and worthwhile to explicitly state the detailed results of the RVB ideas applied to this lattice so as to serve as a reference point for further experiments that are surely forthcoming shortly.

Another reason why the triangular lattice is important is that there exists a very complete and highly nontrivial set of ideas having their origin in the Fractional Quantum Hall physics that have been theoretically applied to the triangular lattice by Kalmeyer and Laughlin [3] Their picture of a $m=2$ FQHE state of interacting hardcore bosons (viz the spin- $1 / 2$ particles ), leads to anyonic particles on doping and to a Meissner like time reversal breaking state. Such a state can be alternately viewed in the language of the flux phases, where Anderson, Shastry and Hristopulous [4] and also Reference [5], showed its equivalence to a flux $\pi / 2$ per triangle state. Systematics of the doping dependence of the optimum flux have not apparently been done, and presumably that is another interesting area to pursue in the present context.

Earlier weak coupling results on superconductivity in the triangular lattice [6] were motivated by experiments on organic superconductors. A recent high temperature expansion study of the t-J model on the triangular lattice estimates the total entropy and magnetic susceptibility as a function of temperature and doping [7].

In the present work, we perform what seems to be a consistent and simple version of RVB theory, one which yields $\mathrm{d}$-wave order for the square lattice [8] and also for the other recently interesting case of $\mathrm{SrCu}_{2}\left(\mathrm{BO}_{3}\right)_{2}$ [9]. Earlier calculations have been done by other authors in a similar spirit to ours, Reference [5] is confined to half filling, and a recent preprint by Baskaran [10] makes some qualitative points that are common to our calculations. Within this version, we evaluate the case of positive as well as negative hoppings since these are so very different in their physical content. Using the particle-hole transformation for fermions, we may define two broad cases of interest:

- Case A: Here we have either

(i) $t>0$ and electron doping, or

(ii) $t<0$ with hole doping, and

- Case B: where we have either

(i) $t>0$ and hole doping, or

(ii) $t<0$ and electron doping.

Here we note that the hamiltonian of the t-J model is written in the standard form :

$$
\mathrm{H}=-t \sum_{<i, j>, \sigma} \mathcal{P} c_{i \sigma}^{\dagger} c_{j \sigma} \mathcal{P}+\mathrm{J} \sum_{<i, j>}\left(\mathbf{S}_{i} \cdot \mathbf{S}_{j}-\frac{n_{i} n_{j}}{4}\right)
$$

where $\mathcal{P}$ stands for the Gutzwiller projection due to large $\mathrm{U}$, and the summation is over nearest neighbors. The notation of hole and electron filling is relative to half filling in the effective one band model for this system, and as usual, $\delta=|1-n|$, where $n$ is the electron concentration. This model incorporates both electronic frustration and spin frustration through the kinetic and the exchange energies. The former favors Nagaoka ferromagnetism for Case A (see later), while the frustrated antiferromagnetic exchange $\mathrm{J}$ competes against it for both cases. The use of an antiferromagnetic exchange is motivated by the observed Curie Weiss susceptibility with a negative Curie Weiss temperature [11], and though surprising in the event of a $98^{0} \mathrm{Co}-\mathrm{O}-\mathrm{Co}$ bond angle, is not 
without precedent, since $\mathrm{CuGeO}_{3}$ with the same bond angle has also an AFM $\mathrm{J}\left(\sim 120^{\circ} \mathrm{K}\right)[12]$.

A first step in the direction of identifying an effective one band model is in the work of Singh [13], whose LDA calculation of the band structure shows that the fermi energy for the case of $\mathrm{NaCo}_{2} \mathrm{O}_{4}$ is in a tight binding like set of states of $\mathrm{t}_{2 g}$ symmetry, in the close proximity of and slightly above a sharp peak in the electronic density of state. We interpret this as an example of Case A(i) above, since the fermi surface for triangular lattice tight binding band structure, $\epsilon(\mathbf{k})=$ $-2 t\left(\cos \left(k_{x}\right)+2 \cos \left(k_{x} / 2\right) \cos \left(\sqrt{3} k_{y} / 2\right)\right.$, gives a density of states (DOS) (see Figure 1) with a prominent peak near the fermi level for $\mathrm{Na}_{0.5} \mathrm{CoO}_{2}$ as well as the extremity of the band, and to the extent that the low energy structures are irrelevant, this matches the LDA DOS [14]. We return to discuss this issue in the summary section. The fiduciary Mott insulating state from where the hole/electron doping is measured, is the case of pure $\mathrm{CoO}_{2}$ on a triangular lattice. This particular system has apparently not been realized experimentally so far due to a lattice structure change.

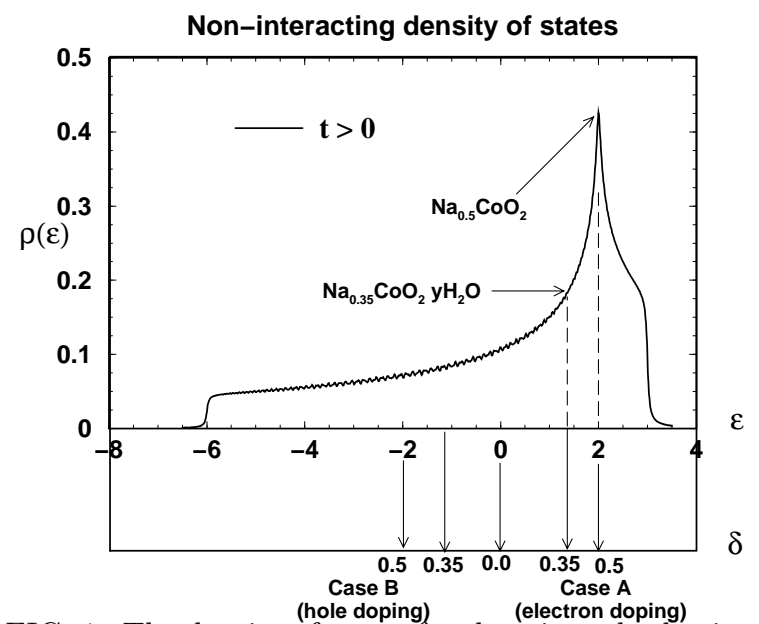

FIG. 1. The density of states for the triangular lattice tight binding hamiltonian, and locations of various systems.

\section{A FEW REMARKS ON FERROMAGNETISM AND THE HALL CONSTANT IN THE TRIANGULAR LATTICE}

Before describing the RVB calculation, we recount a few remarkable features of the triangular lattice Hubbard and t-J model physics, that may be useful in future studies.

\section{A. Ferromagnetism}

Singh has noted that the LDA calculations of $\mathrm{NaCo}_{2} \mathrm{O}_{4}$ show an instability of the paramagnetic state towards a ferromagnetic state. Indeed this is exactly what one expects from the Nagaoka physics on the triangular lattice as shown by Shastry, Krishnamurthy and Anderson [15], who pointed out that while Case B above is highly detrimental to ferromagnetism in the infinite U limit, Case A highly favors the ferromagnetic state. This follows from a stability analysis of the low energy excitations of the state. Ferromagnetism is the fate of the Case A, t- $J$ model at $J=0$ for essentially all fillings. At low dopings, turning on a sufficiently strong antiferromagnetic $J$ removes the Nagaoka instability, for some range of hole doping. We argue below that in this very doping range, the RVB superconducting phase emerges instead. At higher doping, i.e. in the high electron density limit of Case A(i), J becomes irrelevant. For an almost filled band, with the fermi energy near a peak in the DOS (as in transition metal ferromagnetism) the work of Kanamori and Galitskii predicts ferromagnetism. Thus notwithstanding the results of the RVB state, we must expect metallic ferromagnetism in the case when the electron doping is high in Case A(i). The observation of ferromagnetism in $\mathrm{Na}_{0.75} \mathrm{CoO}_{2}$ [16] is consistent with these arguments, while being enigmatic in that the high temperature susceptibility shows a negative Curie Weiss temperature.

\section{B. High Frequency Hall Effect}

A fascinating property of the triangular lattice was noted in Reference [17]. The high frequency Hall constant $R_{H}^{*}$ is amenable to a lattice walk expansion. It can be expressed in terms of loops encircling a flux, and manages to capture the Mott Hubbard aspect of the problem, such as a vanishing "effective Hall carrier density" near half filling on the square lattice. The resulting high frequency Hall constant, in Mott Hubbard systems is not a measure of carrier density, unlike in simple conductors, but encodes complicated correlations of the underlying system. The topology of the lattice plays a critical role in determining this object, since it depends upon the length of the closed loops, and the triangular lattice was noted to be exceptional in having the smallest length of a closed loop, with an odd number of steps namely 3 , leading to a very different behaviour from say the square lattice. A calculation for the triangular lattice in the case of hole doping yields

$$
R_{H}^{*}=-\frac{v}{8|e|} \frac{k_{B} T}{t} \frac{1+\delta}{\delta(1-\delta)}(\text { Cases } \mathrm{A}(\mathrm{ii}) \text { or } \mathrm{B}(\mathrm{i})) .
$$

Here $|e|$ is the magnitude of the electronic charge and $v$ is the physical (three dimensional) unit cell volume containing one cobalt ion, which from Reference [1] may be estimated to be $67.71 \times 10^{-24} \mathrm{~cm}^{3}$ [18]. We remark that this result is computed in the case of hole doping and either sign of hopping " $t$ ", i.e. Case A(ii) or B(i), and 
in comparing with electron doping, $\mathrm{A}(\mathrm{i})$ or $\mathrm{B}(\mathrm{ii})$, one must use the usual rules for particle-hole transformation $t \rightarrow-t$ as well as $\delta=|1-n|$. This leads to the following expression for $R_{H}^{*}$ in the case of electron doping,

$$
R_{H}^{*}=\frac{v}{8|e|} \frac{k_{B} T}{t} \frac{1+\delta}{\delta(1-\delta)}(\operatorname{Cases} \mathrm{A}(\mathrm{i}) \text { or } \mathrm{B}(\mathrm{ii})) .
$$

The expressions in Equations 2 and 3 are valid when temperature, $\mathrm{T}>\mathrm{T}_{\text {degeneracy }} \sim|t|$. Since $\mathrm{T}_{\text {degeneracy }}$ seems so low in these systems, as evinced by the strong CurieWeiss behavior, this result, so remarkable in its absence of saturation in temperature, seems worthwhile to check experimentally. This could also be used to experimentally determine the magnitude as well as sign of " $t$ " for an effective one band system. The distinction between the transport and the high frequency Hall constants is argued to be through a weakly frequency and temperature dependent self energy, and hence it is possible that the transport measurements are also anomalous in the same sense [19].

\section{THE RVB CALCULATION AND ITS DETAILED PREDICTIONS}

\section{A. Mean field equations}

The ' $\mathrm{J}$ ' term in Equation 1 can be re-written as $-\mathrm{J} \sum_{i, j} \mathrm{~b}_{i j}^{\dagger} \mathrm{b}_{i j}$, where the bond operator, $\mathrm{b}_{i j}^{\dagger}=\left(c_{i \uparrow}^{\dagger} c_{j \downarrow}^{\dagger}-\right.$ $\left.c_{i \downarrow}^{\dagger} c_{j \uparrow}^{\dagger}\right) / \sqrt{2}$, is a singlet pair creation operator acting on a pair of sites $i$ and $j$. The RVB mean field calculations are carried out by defining a complex order parameter, $\Delta_{i j}=\left\langle b_{i j}\right\rangle$. On the triangular lattice, we have three different nearest neighbor bonds, one along say $\mathrm{x}$-direction and the other two at an angle of $\pi / 3$ and $2 \pi / 3$ with $\mathrm{x}$ axis. We consider a simple situation where the $\left|\Delta_{i j}\right|=\Delta$ along all bonds, but three different phases are allowed along three kinds of bonds. Since one of the phases can be gauged away, only two (relative) phases are sufficient. We assign zero phase along the x-direction, $\theta$ and $\phi$ along the $\pi / 3$ and $2 \pi / 3$ directions, respectively. In momentum space, this choice of mean field order parameter leads to the following k-space function, $\mathrm{D}(\mathbf{k})$, which carries the order parameter symmetry information.

$$
\begin{aligned}
\mathrm{D}(\mathbf{k})=\cos \left(k_{x}\right)+ & e^{i \theta} \cos \left(k_{x} / 2+\sqrt{3} k_{y} / 2\right) \\
& +e^{i \phi} \cos \left(k_{x} / 2-\sqrt{3} k_{y} / 2\right)
\end{aligned}
$$

The effect of projection of double occupancy on hopping is accounted for by a simple approximation where $t$ is replaced by $t \delta$, with $\delta$ being the hole concentration. Our calculations have been done only for hole doping case, but for both $t>0$ as well as $t<0$ (Cases B(i) and $\mathrm{A}(\mathrm{ii})$ ). The case of electron doping can easily be related to these calculations by particle-hole transformation, as described earlier.
We get two mean field equations, one for $\Delta$ and the other for $\mu$, the chemical potential. These are :

$$
\begin{aligned}
\Delta & =\frac{1}{6 \mathrm{~J} L} \sum_{\mathbf{k}} \frac{\partial \mathrm{E}(\mathbf{k})}{\partial \Delta} \tanh \left(\frac{\beta \mathrm{E}(\mathbf{k})}{2}\right) \\
\delta & =-\frac{1}{L} \sum_{\mathbf{k}} \frac{\partial \mathrm{E}(\mathbf{k})}{\partial \mu} \tanh \left(\frac{\beta \mathrm{E}(\mathbf{k})}{2}\right)
\end{aligned}
$$

Here, $\mathrm{E}(\mathbf{k})=\sqrt{(\epsilon(\mathbf{k})-\mu)^{2}+2 \mathrm{~J}^{2} \Delta^{2}|D(\mathbf{k})|^{2}}, \mathrm{D}(\mathbf{k})$ is given in Equation 4.

\section{B. Order parameter and Quasiparticle spectrum}

We solve Equations 5 and 6 self-consistently for given values of $\delta$ and $t$, and for different choices of $\theta$ and $\phi$. All energies are measured in units of the exchange coupling, J.

First, we perform the computation at $\mathrm{T}=0$, and find the values of $\theta$ and $\phi$, at different hole concentrations, for which the ground state energy is minimum. This fixes the symmetry of the mean field order parameter. At $\delta=0$, the ground state energy is lowest for $(\theta, \phi)=(0, \pm \pi / 2)$ and $(\theta, \phi)=( \pm \pi / 2,0)$, in contrast to the case of $(\theta, \phi)=(2 \pi / 3,4 \pi / 3)$ of Reference [5] which has slightly higher energy. At half filling, the ground state has a lower symmetry of cubic type rather than the six-fold rotational symmetry of the triangular lattice.

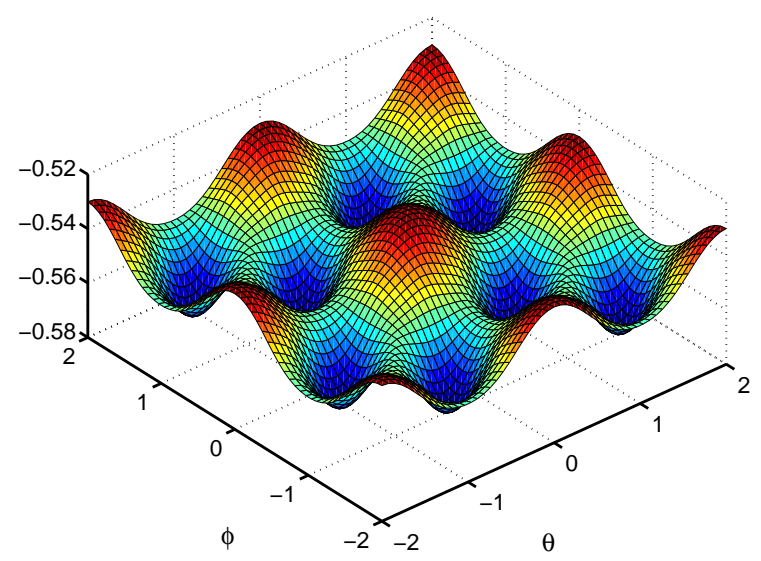

FIG. 2. The mean field energy density surface as a function of the internal phases, $\theta$ and $\phi$ (in units of $\pi$ ) of the order parameter. The minima occur at six corner points of the Brillouin zone.

Away from half filling, the ground state energy is lowest at $(\theta, \phi)=(2 \pi / 3,4 \pi / 3),(4 \pi / 3,2 \pi / 3)$ and $(2 \pi / 3,-2 \pi / 3)$. Three other phase-points, which are related to these via inversion with respect to origin, are equivalent, and together these six minimum energy phase-points reflect the symmetry of the Brillouin zone. 
Figure 2 shows the mean field energy density plotted as a function of $\theta$ and $\phi$. In rest of the calculations, we just choose one of these points to perform the computation.

At $\theta=2 \pi / 3$ and $\phi=4 \pi / 3$, for various values of $t$, the mean field $\Delta$ is computed as a function of hole concentration, as shown in Figure 3. $\Delta$ decreases rapidly for $t>0$ rather than for $t<0$, as the hole concentration is increased. The larger magnitude of $\Delta$ as well as the greater doping range suggests that Case $\mathrm{A}$, that is " $t<0$ and hole doping" or " $t>0$ and electron doping ", presents a more robust case for the RVB state of superconductivity, as compared to the Case B.

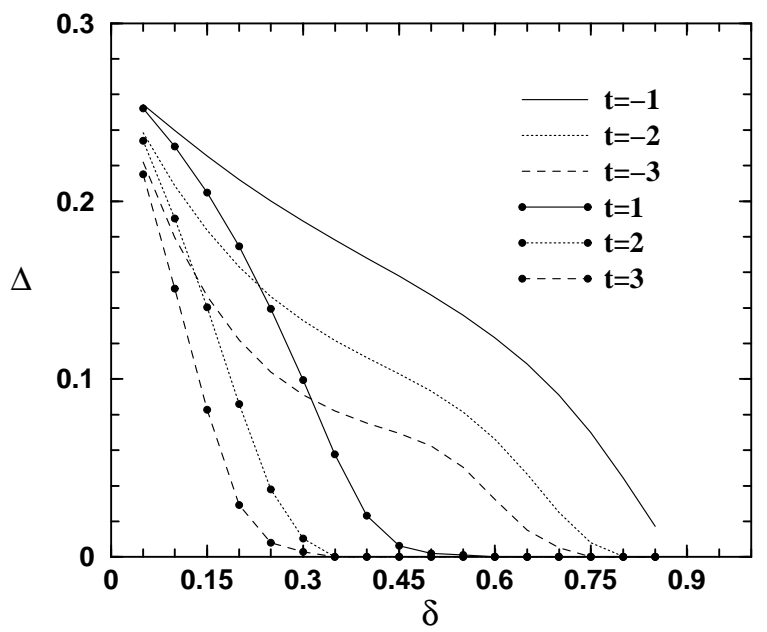

FIG. 3. The mean field $\Delta$ as a function of the hole concentration, $\delta$, for both positive and negative $t$ in units of $\mathrm{J}$.

The results of this low temperature mean field theory should be contrasted to those of the high temperature series results of $\operatorname{Ref}([7])$, who interpret their extrapolated results to imply lower entropy at low temperatures $(k T<|t|)$ for Case B, and hence perhaps greater tendency towards some (non ferromagnetic) ordering. Further work needs to be done to reconcile these findings.

Next we present the quasi-particle dispersion and the quasi-particle density of states from our mean field calculations. These calculation are done for a physically relevant value of $\delta \sim 0.35$ at which superconductivity is observed in $\mathrm{Na}_{x} \mathrm{CoO}_{2} \cdot y \mathrm{H}_{2} \mathrm{O}$ [1]. The cobalt oxide layer alone acts as a half filled system in the effective one band picture, therefore the carrier concentration in excess to the half filled case is just $x$.

Both the quasi-particle dispersion and the density of states, in Figures 4 and 5 respectively, show an energy gap in the spectrum. The reason lies in the fact that function $\mathrm{D}(\mathbf{k})$ is complex. For $(\theta, \phi)=$ $(2 \pi / 3,4 \pi / 3)$, the function $\mathrm{D}(\mathbf{k})=d_{1}-i d_{2}$, where $d_{1}=\cos \left(k_{x}\right)-\cos \left(k_{x} / 2\right) \cos \left(\sqrt{3} k_{y} / 2\right)$ and $d_{2}=$ $\sqrt{3} \sin \left(k_{x} / 2\right) \sin \left(\sqrt{3} k_{y} / 2\right)$. Thus our results are akin to the $d_{x^{2}-y^{2}}+i d_{x y}$ symmetry case in the cuprates.

The modulus of $\mathrm{D}(\mathbf{k})$ is non-zero at all points in the
Brillouin zone, except at six zone corner points, that is $(2 \pi / 3,-2 \pi / 3),(2 \pi / 3,4 \pi / 3)$ etc and the origin. Though $\epsilon(\mathbf{k})-\mu$ has contour of zeros within the Brillouin zone, the chance of six corner points lying on this contour of zeros is almost zero. This point is clear in Figure 6, where $|\mathrm{D}(\mathbf{k})|$ and $\epsilon(\mathbf{k})-\mu$ are plotted along three symmetry directions of the Brillouin zone. The gap for $t=-1$ and -2 is approximately $0.2 \mathrm{~J}$ and $0.1 \mathrm{~J}$, respectively.

The gap $(\sim|\Delta|)$ decreases with increasing $|t|$ as already noted. We mention that the quasi-particle spectrum is gapped for $t>0$ as well, but the gap is very small around the doping of our interest. In fact for reasonable values of $t$ (say, 3) it is zero, precisely because $\Delta=0$. Thus, Case B does not favor RVB solution for large dopings, say 0.3 or beyond.

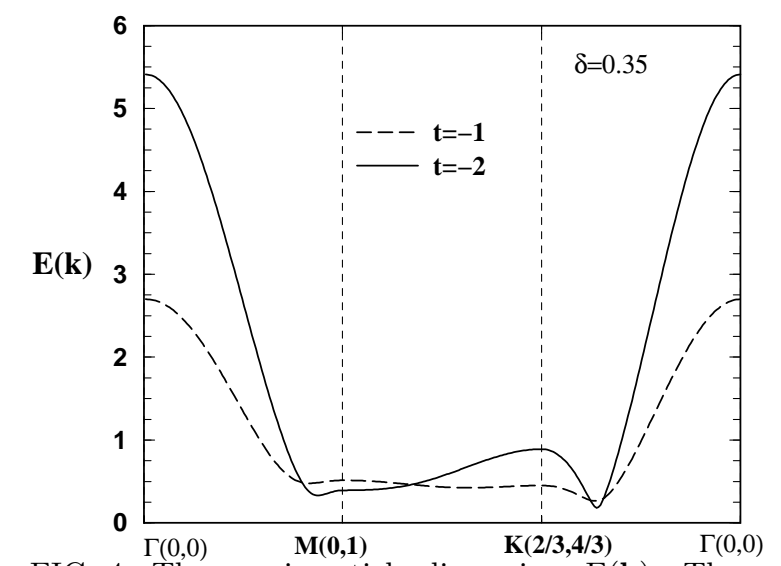

FIG. 4. The quasi-particle dispersion, $\mathrm{E}(\mathbf{k})$. The coordinates of $\Gamma, \mathrm{M}$ and $\mathrm{K}$ symmetry points in the Brillouin zone are given in units of $\pi$. The coordinates $\left(k_{1}, k_{2}\right)$ given here are such that $k_{1}=k_{x}$ and $k_{2}=\left(k_{x}+\sqrt{3} k_{y}\right) / 2$, where $k_{x}$ and $k_{y}$ are the usual k-space variables along $x$ and $y$ directions.

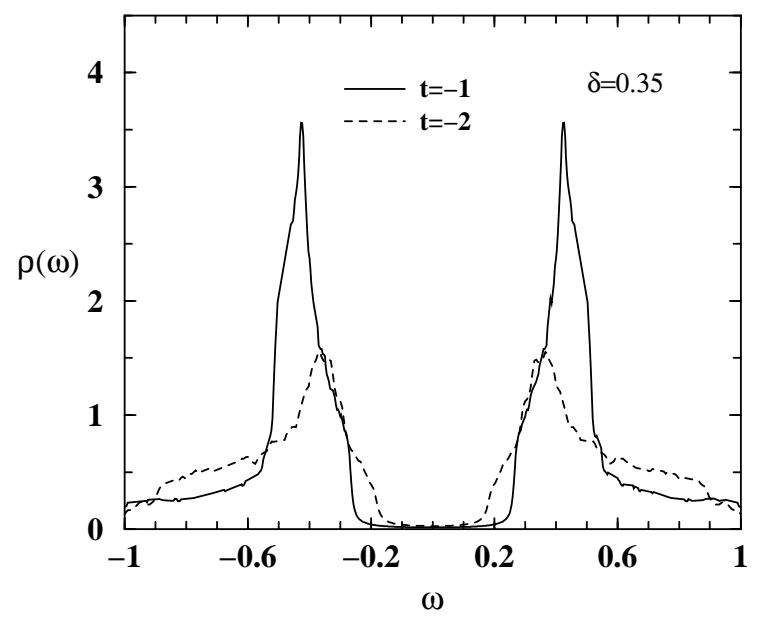

FIG. 5. The quasi-particle density of states.

We have also calculated temperature at which the mean field order parameter $\Delta$ vanishes for different values of $\delta$. It helps us understand the broad nature of thermo- 
dynamic phase diagram in T- $\delta$ plane. Figure 7 shows the transition temperatures for different hole concentrations for which $\Delta$ vanishes.

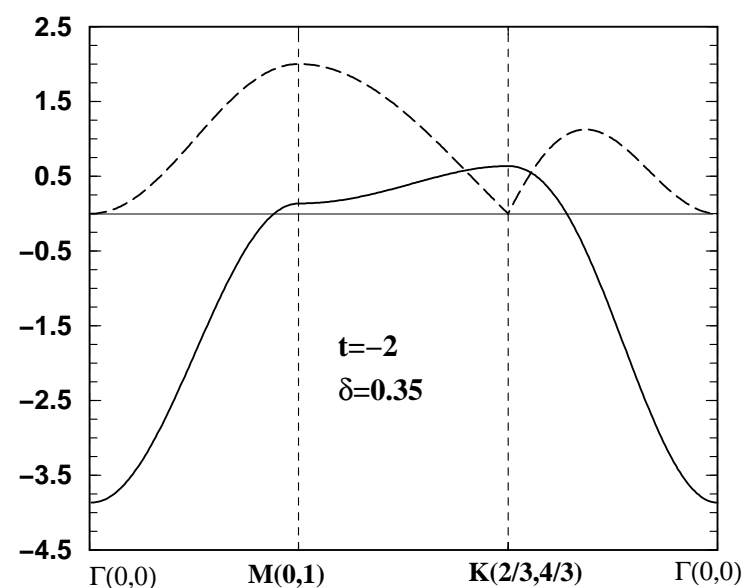

FIG. 6. The dashed line is for the $|\mathrm{D}(\mathbf{k})|$ and the solid line is for $(\epsilon(\mathbf{k})-\mu) / 2 t \delta$.

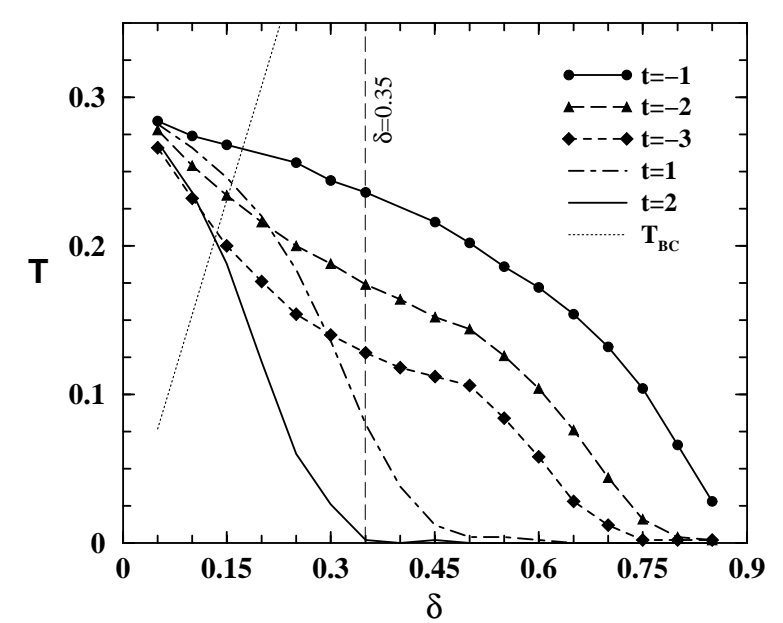

FIG. 7. $\mathrm{T}$ is in units of J. The case with $t>0$ for $\delta \sim 0.35$ is not very robust for the RVB mean field theory whereas $t<0$ is favourable.

The question of RVB superconductivity needs a little more care than what we have given so far. The mean field $\Delta$, though a pairing order parameter, doesn't by itself imply superconductivity. For example at half filling $\Delta$ is non-zero, but it is insulating. The identification of the superconducting phase in $\mathrm{T}-\delta$ diagram can, however, be done within the frame work of the slave boson approach. This approach has been quite popular in the RVB theories of t-J model on square lattice [22]. Here the physical electron operator, $c_{\sigma}^{\dagger}=f_{\sigma}^{\dagger} b$, where $f$ is the spin- $1 / 2$ neutral fermion, and $b$ is the spin- 0 charged boson, and the projection of double occupancy is in-built into the construction.

The superconducting order parameter $\left\langle c^{\dagger} c^{\dagger}\right\rangle \sim$ $\langle b b\rangle\left\langle f^{\dagger} f^{\dagger}\right\rangle$ is a product of spin pairing order parameter and a bose condensation factor. What we have got from the mean field calculation is essentially the spin-pairing order parameter. The true superconducting order parameter is $\Delta_{S C} \sim F_{B} \Delta$, where $\Delta$ is the mean field order parameter. The bose condensation temperature for $b$ bosons (for $F_{B}$ to be non-zero) needs to be estimated separately. That region of T- $\delta$ diagram, where both $F_{B}$ as well $\Delta$ are non-zero, can be interpreted as the RVB superconducting phase. There are three more typical regions according to this interpretation : (1) Spin-gap : $\Delta \neq 0$ and $F_{B}=0$, (2) Strange metal : $\Delta=F_{B}=0$, and (3) Normal metal : $\Delta=0$ and $F_{B} \neq 0$. With this qualitative picture in mind, we will present a rough phase diagram for the cobalt oxide superconductors.

Let us briefly mention how we estimate the bose condensation temperature, $\mathrm{T}_{B C}$, for the bosons. There are other ways [22], but we discuss our roughly equivalent prescription. The $b$ bosons, at the level of mean field decoupling, will effectively have the same band structure as that of the tight binding electrons on triangular lattice. Therefore, the $\mathrm{T}_{B C}$, is defined as a temperature at which the boson chemical potential tends to be the band's bottom. Since free bosons can not condense in two dimensions, we will take the case of three dimensional band structure with a large c-axis anisotropy. Around the band's bottom, the energy dispersion can be approximated as $c^{*}\left(k^{2}+k_{z}^{2} / \gamma\right)$. The curvature, $c^{*}$, is related to the two dimensional density of states at the band edge, $\rho^{*}$ as : $c^{*} \approx \frac{1}{4 \pi \rho^{*}}$. Now, using the fact that free boson can condense in three dimensions, $\delta=\frac{1}{L} \sum_{\mathbf{k}} 1 /\left(e^{\beta_{B C}\left(\epsilon(\mathbf{k})-\epsilon_{\min }\right)}-1\right)$. Simplifying it for $\gamma \gg \pi / 4$, we get

$$
T_{B C} \approx \frac{\delta}{\rho^{*}} \frac{1}{2+\log (4 \gamma / \pi)}
$$

Since the anisotropy affects only logarithmically, we can safely take some large value for $\gamma \sim 100$, especially when the real system is a good quasi two dimensional system.

\section{Phase Diagram}

Here, we propose a rough phase diagram for triangular lattice, layered cobalt oxide materials over a wide range of doping based upon our calculation in the scheme of Case A(ii) as shown in Figure 8. This phase diagram encompasses a number of interesting phases, including the recently observed superconducting phase. We will briefly describe each of the labelled region in Figure 8. 


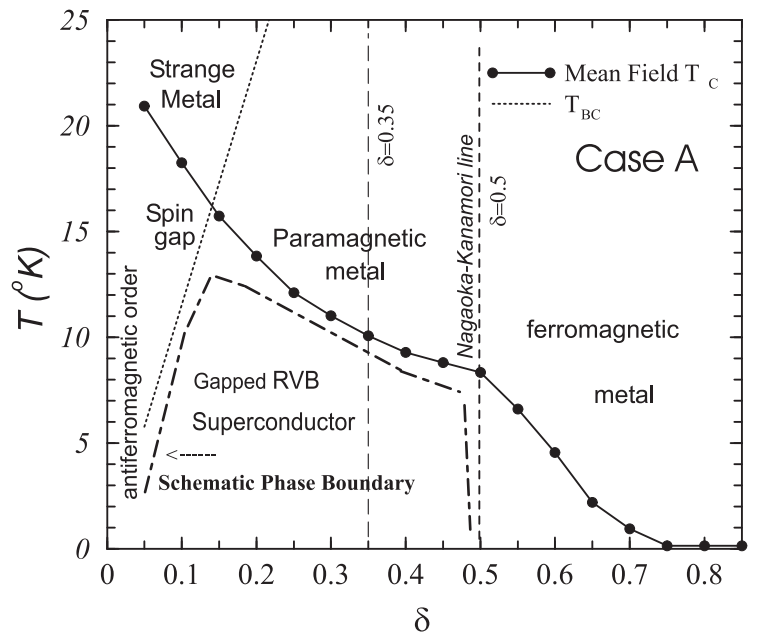

FIG. 8. The mean field phase diagram for $t=-3 \mathrm{~J}$ and $(\theta, \phi)=(2 \pi / 3,4 \pi / 3)$.

The exchange coupling $\mathrm{J}$ is estimated from the high temperature susceptibility data [11] for $\mathrm{Na}_{0.5} \mathrm{CoO}_{2}$. Its Curie Weiss temperature is approximately $-118 \mathrm{~K}$ which gives $\mathrm{J} \sim 79 \mathrm{~K}$. For doping $\delta=0.35$, the $\mathrm{T}_{C}$ for transition into an RVB superconducting state is approximately $10 \mathrm{~K}$, compared to the measured $\mathrm{T}_{C} \sim 5 \mathrm{~K}[1]$. An important prediction for the superconducting phase is the existence of an energy gap in the quasi-particle spectrum. It arises because the superconducting order parameter is complex, with relative internal phases $2 \pi / 3$ and $4 \pi / 3$ thus avoiding the possibility of generic vanishing of the gap along lines or points in the momentum space. The optimal doping seems to be around 0.15 .

On the higher side of the doping $\delta \gtrsim 0.5$ in Figure 8, we expect the RVB superconducting state to become unstable in favor of a ferromagnetic metallic state where either the Nagaoka or the Kanamori ferromagnetism plays a dominant role. We call $\delta=0.5$ (electron concentration, $n=1.5$ ) as the Nagaoka-Kanamori line, as the rough location where the Nagaoka physics of the doped Mott state transmutes into the Brueckner type Kanamori physics of multiple scattering in an almost filled band. The precise location of the phase boundary between the superconducting and the ferromagnetic state is difficult to predict from the present calculation, since the RVB mean field theory is not particularly accurate in getting the absolute numbers for various states. We present only a guide to the eye in our phase diagram. At sufficiently high temperatures, we expect ferro-metal to para-metal transition across the Nagaoka-Kanamori line which can be estimated from the spin stiffness of the quasi 2-d ferromagnet. Apart from the above mentioned important phases, one may expect spin-gapped phase in the low doping, low temperature regime, since that is where exchange interaction will play more decisive role. The three sublattice antiferromagnetic phase of the triangular lattice AFM is likely to persist for small doping. We also expect a strange metallic phase to exist at moderate dop- ing, but only at higher temperatures.

We next also present, in Figure 9, the mean field phase diagram for Case B(i). Though Case B is less favourable than Case A, it does yet achieve the RVB superconducting phase for small values of $t(\sim \mathrm{J}$ or smaller; see Figure 7). Since the value of $|t|$ in these cobaltates is believed to be rather low, it seems appropriate to record the results for Case B also.

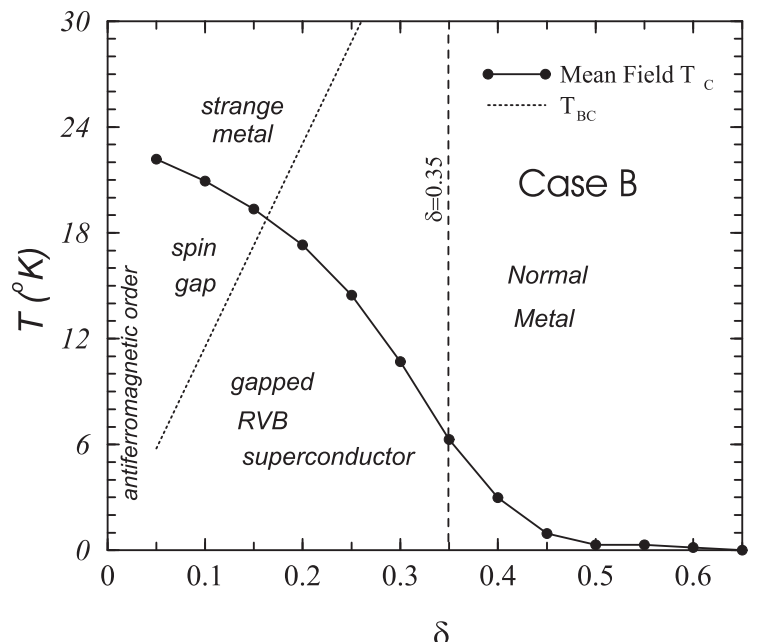

FIG. 9. The mean field phase diagram for $t=\mathrm{J}$ and $(\theta, \phi)=(2 \pi / 3,4 \pi / 3)$.

This phase diagram is a simpler version of the mean field phase diagram for Case A(ii), with the symbols having the same meaning. The possibility of a ferromagnetic phase does not arise in this case, leading to its simplicity. Here we find $\mathrm{T}_{C} \sim 6 \mathrm{~K}$ at 0.35 hole concentration. The optimal doping and the maximum $\mathrm{T}_{C}$ are similar to those of Case A, but $\mathrm{T}_{C}$ falls off more rapidly for $\delta \geq 0.15$.

\section{SUMMARY}

To summarize the work presented here, we have performed an RVB mean field theory on the triangular lattice inspired by the recent discovery of the superconductivity in cobalt oxide layered materials.

Our use of a single band model in the present context needs a word of justification. The LDA band structure for $\mathrm{Na}_{0.5} \mathrm{CoO}_{2}[13,14]$ indicates the possibility of a competition between two bands for the fermi level on moving away from that doping in either direction. One would conclude that $\delta_{c} \sim 0.5$ if the bands are held rigidly under doping, since at this filling the fermi level tangents the (filled) band emanating from the $\Gamma$ point in Fig. 1 of Reference [13]. The systems at different dopings would have their fermi levels in different bands. These would then correspond to (1) Case A(i) for $\delta<\delta_{c}$ i.e. electron like and (2) $\mathrm{B}(\mathrm{ii})$ for $\delta>\delta_{c}$, i.e. hole like. A "one band model" appears to be justifiable provided one is sufficiently far from $\delta_{c}$. In view of the uncertainty in the ex- 
act location of $\delta_{c}$, we have presented the results for both signs of $\mathrm{t}$ above. While photoemission may shed light on this issue, its interpretation needs caution in view of the remarkable possibility that the observed renormalized fermi surface in strongly correlated systems could differ drastically in shape from the bare fermi surface, that has emerged from recent numerical work $[20,21]$. Our concern in this work is with the "bare fermi surface", i.e. with the sign of the bare $t$.

We argue that the superconducting material $\delta=0.35$ corresponds to an effective one band case with $t>0$ and electron doping, i.e. A(i). This is logical since a filling of $\delta=0.35$ seems far enough from any reasonable $\delta_{c}$. Several detailed results are presented. We find the symmetry of the superconducting order parameter, and predict the existence of an energy gap. We also present an approximate phase diagram for the cobalt oxide superconductor in the $\mathrm{T}-\delta$ plane.

In the more interesting Case A, there is a competition between ferromagnetic order and superconductivity. Our low temperature RVB superconducting phase basically arises in the doping range where Nagaoka ferromagnetism is suppressed by the antiferromagnetic J. The superconductivity found here may thus be viewed as arising from what may be termed total frustration, i.e. from the competition between competing terms, the frustrated electronic motion that weakly prefers Nagaoka ferromagnetism, and the frustrated spin exchange that prefers either no long ranged order (i.e. a short ranged RVB type state) or a doping weakened three sublattice order.

One major assumption in this work is that the scale of $t$ is not too different from $\mathrm{J}$ which is estimated to be around $79 \mathrm{~K}$. This estimate is at odds with the LDA estimate of the band width by two orders of magnitude, and thus we cannot claim to have "explained" the low degeneracy temperature scale, we have merely assumed it and worked out the consequences for other properties. Indeed the emergence of a low energy scale in these systems seems to be a central problem of the cobaltates.

Finally we have presented our results for the high frequency Hall constant $R_{H}^{*}$. This is predicted to grow linearly with temperature without saturation, and the slope depends in a known way on $t$ and the filling. The filling dependence $\frac{1+\delta}{\delta(1-\delta)}$ should be readily testable. The explicit expressions enable one to extract the hopping matrix element $t$. The unusual behaviour of the Hall constant is a special property of the triangular lattice, having to do with its unique topology of smallest length of closed loops, with odd length. Our results are valid for high temperatures $\left(k_{B} \mathrm{~T} \gg|t|\right)$. Remarkably enough, this condition appears easy to fulfill in the cobaltates, and hence it should be possible to utilize these results to extract basic parameters for the system from high frequency Hall experiments. Our result might also be useful in interpreting transport Hall data.

BK acknowledges CSIR for financial support. BSS was supported in part by an Indo French grant IFC$\mathrm{PAR} / 2404.1$

[1] K. Takada, H. Sakurai, E. Takayama-Muromachi, F. Izumi, R. A. Dilanian and T. Sasaki, Nature 422, 53 (2003)

[2] P. W. Anderson, Mat. Res. Bull. 8, 153 (1973)

P. W. Anderson, Science 235, 1196 (1987)

[3] V. Kalmeyer and R. B. Laughlin, Phys. Rev. Lett. 59, 2095 (1987)

[4] P. W. Anderson, B. S. Shastry and D. Hristopulos, Phys. Rev. B 40, 8939 (1989)

[5] T. K. Lee and S. Feng, Phys. Rev. B 41, 11110 (1990)

[6] M. Vojta and E. Dagotto, Phys. Rev. B 59, R713 ( 1999).

[7] T. Koretsune and M. Ogata, Phys. Rev. Letts. 89, 116401 ( 2002).

[8] G. Kotliar and J. Liu, Phys. Rev. B 38, 5142 (1988)

[9] B. S. Shastry and B. Kumar, Prog. Theor. Phys., Supplement 145, 1 (2002)

[10] G. Baskaran, cond-mat/0303649, here the assignment of the sign of the hopping element " $t$ " seems to be the opposite to ours, namely to Case B(ii).

[11] R. Ray, A. Ghoshray, K. Ghoshray, S. Nakamura, Phys. Rev. B 59, 9454 (1999)

[12] M. Nishi, O. Fujita and J. Akimitsu, Phys. Rev. B 50, 6508 (1994)

[13] D. Singh, Phys. Rev. B 61, 13397 (2000)

[14] Our assignment of the sign of " $t$ " leads to an electron like fermi surface surrounding the $\Gamma$ point, which might be ascribable to the electron like band in Figure 1 of Reference [13] having a minimum at the $\Gamma$ point. This is reasonable if remember that the electron count for the superconducting samples $x=0.35$ forces the fermi level to dip below the value for the $x=0.5$ case reported in Reference [13] which has a hole like fermi surface surrounding the $\Gamma$ point.

[15] B. S. Shastry, H. R. Krishnamurthy and P. W. Anderson, Phys. Rev. B 41, 2375 (1990)

[16] T. Motohashi, R. Ueda, E. Naujalis, T. Tojo, I. Terasaki, T. Atake, M. Karppinen and H. Yamauchi, Phys. Rev. B 67, 064406 ( 2003).

[17] B. S. Shastry, B. I. Shraiman and R. R. P. Singh, Phys. Rev. Lett. 70, 2004 (1993)

[18] We get lattice parameters $a=2.823 \AA$ and $c=$ $19.621 \AA$ from Reference [1]. The physical cell volume containing one cobalt ion, $v=\frac{1}{2} \frac{\sqrt{3} a^{2} c}{2}$. The factor of $1 / 2$ is there because each crystallographic unit cell of $\mathrm{Na}_{x} \mathrm{CoO}_{2} \cdot y \mathrm{H}_{2} \mathrm{O}$ contains two $\mathrm{CoO}_{2}$ layers, each contributing one cobalt ion.

[19] With an absolute scale measurement of $R_{H}^{*}$, it should be possible to obtain not only the sign of " $t$ ", but also its magnitude. Optical Hall constant measurements may be roughly guessed at from the transport measurements. 
[20] A. Himela and M. Ogata, Phys. Rev. Letts. 854345 ( 2000).

[21] W. O. Puttika, M. U. Luchini and R. R. P. Singh, Phys.
Rev. Letts. 81, 2966 ( 1998)

[22] M. U. Ubbens and P. A. Lee, Phys. Rev. B 49, 6853 (1994). 